\title{
1 : 3 Resonance and Chaos in a Discrete Hindmarsh-Rose Model
}

\author{
Bo Li and Zhimin He \\ School of Mathematics and Statistics, Central South University, Changsha, Hunan 410083, China \\ Correspondence should be addressed to Zhimin He; hezhimin@mail.csu.edu.cn
}

Received 10 July 2014; Accepted 30 November 2014; Published 17 December 2014

Academic Editor: Zhidong Teng

Copyright (C) 2014 B. Li and Z. He. This is an open access article distributed under the Creative Commons Attribution License, which permits unrestricted use, distribution, and reproduction in any medium, provided the original work is properly cited.

1:3 resonance of a two-dimensional discrete Hindmarsh-Rose model is discussed by normal form method and bifurcation theory. Numerical simulations are presented to illustrate the theoretical analysis, which predict the occurrence of a closed invariant circle, period-three saddle cycle, and homoclinic structure. Furthermore, it also displays the complex dynamical behaviors, especially the transitions between three main dynamical behaviors, namely, quiescence, spiking, and bursting.

\section{Introduction}

When the bifurcation problem of a system

$$
x \longmapsto f(x, \alpha), \quad x \in \mathbb{R}^{n}, \alpha \in \mathbb{R}^{k},
$$

is studied by normal form method, researchers are often likely to compute the following two conditions: nondegeneracy conditions and transversality conditions, in which the derivatives of $f(x, \alpha)$ with respect to the variables $x$ and the parameters $\alpha$ are involved, respectively. If some of the nondegeneracy and transversality conditions for the oneparameter bifurcations would be violated, the two-parameter bifurcations can also happen [1-3]. In this case, one can obtain cusp, generalized flip, and Chenciner bifurcation [2]. In the other case, extra multipliers can approach the unit circle for discrete dynamical system, thus changing the dimension of the center manifold $W^{c}$. There are eleven kinds of two-parameter bifurcations for discrete dynamical system listed by Kuznetsov (see Section 4 in [2]).

In this paper, we focus on $1: 3$ resonance, which is less discussed in the existing papers. When Neimark-Sacker bifurcation is considered, the case that $\theta_{0}=2 \pi / 3$ can lead to $1: 3$ resonance. One can find more information in [1-3] and references cited therein.

In 1982, Hindmarsh and Rose [4] described a two-variable model of the action potential which is a modification of Fitzhugh's B.v.P model in [5] and explained how the close proximity of the nullclines can be exploited to give a qualitative explanation for burst generation. The Hindmarsh-Rose model is known to reproduce all dynamical behaviors, such as quiescence, spiking, bursting, irregular spiking, and irregular bursting $[4,6]$. Bifurcation analysis is examined once more in the past, with respect to one or two bifurcation parameters [7-11]. Local bifurcations and global bifurcations are also analysed and these bifurcation phenomena can be used to explain the transitions between the dynamical behaviors. For example, the transition between spiking and bursting in the model can be understood by homoclinic bifurcations [12, 13]. More information on bifurcation can be found in $\left[1,4,8_{-}^{-}\right.$ 11, 14-22].

Recently, X. Liu and S. Liu [8] discussed the codimension2 bifurcations of the following two-dimensional HindmarshRose model:

$$
\begin{aligned}
& \frac{d x}{d t}=y-a x^{3}+b x^{2}, \\
& \frac{d y}{d t}=-c-d x^{2}-y,
\end{aligned}
$$

where $x$ represents the membrane, $y$ is recovery variable, and $a, b, c$, and $d$ are positive parameters. Model (2) can describe the transitions between the above five dynamical behaviors, that is, quiescence, spiking, bursting, irregular spiking, and irregular bursting. More related works can be found in $[4,7,9-$ 13, 17-21, 23-26]. 
Applying the forward Euler method to model (2), we obtain the following discrete-time Hindmarsh-Rose system:

$$
\left(\begin{array}{l}
x \\
y
\end{array}\right) \longmapsto\left(\begin{array}{c}
x+\delta\left(y-a x^{3}+b x^{2}\right) \\
y-\delta\left(c+d x^{2}+y\right)
\end{array}\right),
$$

where $\delta>0$ is the step size. In [21,27], we proved that map (3) possesses flip bifurcation, Neimark-Sacker bifurcation, and $1: 1,1: 2$, and $1: 4$ resonance. The aim of this paper is to prove that this discrete model possesses the $1: 3$ resonance. The method we used is based on the normal form method and bifurcation theory of discrete dynamical system (see Kuznetsov, Sections 4 and 9 in [2]).

This paper is organized as follows. In Section 2, we present the existence and local stability of fixed points for map (3). In Section 3, we show that there exist some values of parameters such that map (3) undergoes 1:3 resonance. In Section 4, we present numerical simulations, which not only illustrate our results with the theoretical analysis but also exhibit complex dynamical behaviors. Finally, a brief discussion is given in Section 5 .

\section{Local Dynamics for Fixed Points of Map (3)}

The fixed points of map (3) satisfy the following equations:

$$
\begin{gathered}
y-a x^{3}+b x^{2}=0 \\
c+d x^{2}+y=0
\end{gathered}
$$

So $x^{*}$ is the root of the following equation:

$$
\begin{gathered}
a x^{3}+(d-b) x^{2}+c=0, \\
y^{*}=-c-d x^{* 2} .
\end{gathered}
$$

Using the Cardan formula (see [28]), we get the following results (see also $[8,21]$ ).

Lemma 1. (1) If $27 a^{2} c-4(b-d)^{3}>0$, then map (3) has a unique fixed point $E_{11}\left(x_{11}, y_{11}\right)$, where $x_{11}<\min \{0,2(b-$ d) $/ 3 a\}$.

(2) If $27 a^{2} c-4(b-d)^{3}=0$, then map (3) has two fixed points $E_{21}\left(x_{21}, y_{21}\right)$ and $E_{22}\left(x_{22}, y_{22}\right)$, where $x_{21}<0<x_{22}=$ $2(b-d) / 3 a$.

(3) If $27 a^{2} c-4(b-d)^{3}<0$, then map (3) has three different fixed points, $E_{3 i}\left(x_{3 i}, y_{3 i}\right)(i=1,2,3)$, where $x_{31}<0<x_{32}<$ $2(b-d) / 3 a<x_{33}$.

The stability of these fixed points can be found in [21]. In this paper, we focus on the existence and bifurcation analysis of $1: 3$ resonance. Here, we would like to give the bifurcation set of $1: 3$ resonance.

The Jacobian matrix of map (3) at the fixed point $\left(x^{*}, y^{*}\right)$ is given by

$$
J\left(x^{*}, y^{*}\right)=\left(\begin{array}{cc}
1+\delta\left(-3 a x^{* 2}+2 b x^{*}\right) & \delta \\
-2 \delta d x^{*} & 1-\delta
\end{array}\right),
$$

and the corresponding characteristic equation of the Jacobian matrix $J\left(x^{*}, y^{*}\right)$ can be written as

$$
F(\lambda):=\lambda^{2}-(2+G \delta) \lambda+\left(1+G \delta+H \delta^{2}\right)=0,
$$

where

$$
G=-3 a x^{* 2}+2 b x^{*}-1, \quad H=3 a x^{* 2}-2 b x^{*}+2 d x^{*} .
$$

It is easy to get that two eigenvalues of $J\left(x^{*}, y^{*}\right)$ are

$$
\lambda_{1,2}=1+\frac{\delta}{2}\left(G \pm \sqrt{G^{2}-4 H}\right)
$$

Further, if $\delta=-3 / G$ and $G^{2}=3 H$, then we have $\lambda_{1,2}=$ $( \pm \sqrt{3} i-1) / 2$.

Here, we present the bifurcation set of $1: 3$ resonance as follows:

$$
\begin{aligned}
& F=\{(a, b, c, d, \delta): \\
& \left.\quad \delta=-\frac{3}{G}, d=\frac{(2 G+3)^{2}+3}{24 x^{*}}, \delta, a, b, c, d>0\right\} .
\end{aligned}
$$

It is obvious to find that $G<0$ and $H>0$ from the bifurcation set. Hence, the $1: 3$ resonance only can occur at $E_{11}\left(x_{11}, y_{11}\right), E_{21}\left(x_{21}, y_{21}\right), E_{31}\left(x_{31}, y_{31}\right)$, and $E_{33}\left(x_{33}, y_{33}\right)$. In the following, we present our discussions for $E_{11}\left(x_{11}, y_{11}\right)$. The similar arguments can be undertaken at the fixed points $E_{21}\left(x_{21}, y_{21}\right), E_{31}\left(x_{31}, y_{31}\right)$, and $E_{33}\left(x_{33}, y_{33}\right)$.

\section{1 :3 Resonance}

In this section, we show that there exist some values of parameters such that map (3) undergoes 1:3 resonance by using bifurcation theory [1-3]. Here, the step sizes $\delta$ and $d$ are considered as bifurcation parameters to present bifurcation analysis at the fixed point $E_{11}\left(x_{11}, y_{11}\right)$.

We discuss the 1:3 resonance of map (3) at $E_{11}\left(x_{11}, y_{11}\right)$ when the parameters vary in a small neighborhood of $F$. Taking parameters $\left(a, b, c, d_{0}, \delta_{0}\right)$ arbitrarily from $F$, we consider map (3) with $\left(a, b, c, d_{0}, \delta_{0}\right)$, which is described by

$$
\left(\begin{array}{l}
x \\
y
\end{array}\right) \longmapsto\left(\begin{array}{c}
x+\delta_{0}\left(y-a x^{3}+b x^{2}\right) \\
y-\delta_{0}\left(c+d_{0} x^{2}+y\right)
\end{array}\right) .
$$

The eigenvalues of map (11) at the fixed point $E_{11}\left(x_{11}, y_{11}\right)$ are $\lambda_{1,2}=( \pm \sqrt{3} i-1) / 2$.

Now, we consider a perturbation of map (11) as follows:

$$
\left(\begin{array}{l}
x \\
y
\end{array}\right) \longmapsto\left(\begin{array}{c}
x+\delta\left(y-a x^{3}+b x^{2}\right) \\
y-\delta\left(c+d x^{2}+y\right)
\end{array}\right),
$$

where $\left|\delta-\delta_{0}\right|,\left|d-d_{0}\right| \ll 1$ which are small perturbation parameters.

Let $u=x-x_{11}$ and $v=y-y_{11}$. Then we transform the fixed point $E_{11}\left(x_{11}, y_{11}\right)$ to the origin and map (12) becomes

$$
\left(\begin{array}{l}
u \\
v
\end{array}\right) \longmapsto\left(\begin{array}{c}
\left(1+\delta a_{11}\right) u+\delta v+\delta a_{13} u^{2}-\delta a u^{3} \\
\delta d a_{21} u+(1-\delta) v-\delta d u^{2}
\end{array}\right),
$$


where

$$
\begin{gathered}
a_{11}=-3 a x_{11}^{2}+2 b x_{11}, \quad a_{13}=-3 a x_{11}+b, \\
a_{21}=-2 x_{11} .
\end{gathered}
$$

Map (13) can be denoted as

$$
\left(\begin{array}{l}
u \\
v
\end{array}\right) \longmapsto A(\delta, d)\left(\begin{array}{l}
u \\
v
\end{array}\right)+F(u, v, \delta, d),
$$

where

$$
\begin{gathered}
A(\delta, d)=\left(\begin{array}{cc}
1+\delta a_{11} & \delta \\
\delta a_{21} & 1-\delta
\end{array}\right), \\
F(u, v, \delta, d)=\left(\begin{array}{c}
\delta a_{13} u^{2}-\delta u^{3} \\
-\delta d u^{2}
\end{array}\right) .
\end{gathered}
$$

In the following, we will present our analysis in the critical case. It is easy to find the eigenvalues of $A\left(\delta_{0}, d_{0}\right)$ and their corresponding eigenvector $q\left(\delta_{0}, d_{0}\right) \in \mathbb{C}^{2}$ as follows:

$$
A\left(\delta_{0}, d_{0}\right) q\left(\delta_{0}, d_{0}\right)=\frac{\sqrt{3} i-1}{2} q\left(\delta_{0}, d_{0}\right) .
$$

Here, we also introduce the adjoint eigenvector $p\left(\delta_{0}, d_{0}\right) \in \mathbb{C}^{2}$, satisfying

$$
A\left(\delta_{0}, d_{0}\right)^{T} p\left(\delta_{0}, d_{0}\right)=-\frac{\sqrt{3} i+1}{2} p\left(\delta_{0}, d_{0}\right),
$$

which is normalized according to

$$
\left\langle p\left(\delta_{0}, d_{0}\right), q\left(\delta_{0}, d_{0}\right)\right\rangle=1,
$$

where $\langle\cdot, \cdot\rangle$ means the standard scalar product in $\mathbb{C}^{2}:\langle p, q\rangle=$ $\bar{p}_{1} q_{1}+\bar{p}_{2} q_{2}$.

Now any vector $X=(u, v)^{T} \in \mathbb{R}^{2}$ can be represented in the form

$$
X=z q\left(\delta_{0}, d_{0}\right)+\bar{z} \overline{q\left(\delta_{0}, d_{0}\right)}, \quad z \in \mathbb{C} .
$$

From the above equation, we have

$$
\begin{aligned}
& \left\langle p\left(\delta_{0}, d_{0}\right), X\right\rangle \\
& \quad=\left\langle p\left(\delta_{0}, d_{0}\right), z q\left(\delta_{0}, d_{0}\right)+\bar{z} \overline{q\left(\delta_{0}, d_{0}\right)}\right\rangle \\
& \quad=z\left\langle p\left(\delta_{0}, d_{0}\right), q\left(\delta_{0}, d_{0}\right)\right\rangle+\bar{z}\left\langle p\left(\delta_{0}, d_{0}\right), \overline{q\left(\delta_{0}, d_{0}\right)}\right\rangle .
\end{aligned}
$$

Since

$$
\begin{aligned}
\left\langle p\left(\delta_{0}, d_{0}\right), \overline{q\left(\delta_{0}, d_{0}\right)}\right\rangle & =\left\langle p\left(\delta_{0}, d_{0}\right), \frac{1}{\bar{\lambda}} A \overline{q\left(\delta_{0}, d_{0}\right)}\right\rangle \\
& =\frac{1}{\bar{\lambda}}\left\langle A^{T} p\left(\delta_{0}, d_{0}\right), \overline{q\left(\delta_{0}, d_{0}\right)}\right\rangle \\
& =\frac{\lambda}{\bar{\lambda}}\left\langle p\left(\delta_{0}, d_{0}\right), \overline{q\left(\delta_{0}, d_{0}\right)}\right\rangle,
\end{aligned}
$$

where $\lambda=(\sqrt{3} i-1) / 2$, we get

$$
\left(1-\frac{\lambda}{\bar{\lambda}}\right)\left\langle p\left(\delta_{0}, d_{0}\right), \overline{q\left(\delta_{0}, d_{0}\right)}\right\rangle=0,
$$

which implies that

$$
\left\langle p\left(\delta_{0}, d_{0}\right), \overline{q\left(\delta_{0}, d_{0}\right)}\right\rangle=0 .
$$

Using (19), (21), and (24), we have

$$
z=\left\langle p\left(\delta_{0}, d_{0}\right), X\right\rangle .
$$

From (21) and (25), we get

$$
\begin{aligned}
z_{n+1}=\left\langle p\left(\delta_{0}, d_{0}\right), X_{n+1}\right\rangle \\
=\left\langle p\left(\delta_{0}, d_{0}\right), A\left(\delta_{0}, d_{0}\right) X_{n}+F\left(X_{n}, \delta_{0}, d_{0}\right)\right\rangle \\
=\left\langle p\left(\delta_{0}, d_{0}\right), A\left(\delta_{0}, d_{0}\right)\left(z_{n} q\left(\delta_{0}, d_{0}\right)+\overline{z_{n}} \overline{q\left(\delta_{0}, d_{0}\right)}\right)\right. \\
\left.\quad+F\left(z_{n} q\left(\delta_{0}, d_{0}\right)+\overline{z_{n}} \overline{q\left(\delta_{0}, d_{0}\right)}, \delta_{0}, d_{0}\right)\right\rangle \\
=\left\langle p\left(\delta_{0}, d_{0}\right), z_{n} A\left(\delta_{0}, d_{0}\right) q\left(\delta_{0}, d_{0}\right)+\overline{z_{n}} A \overline{q\left(\delta_{0}, d_{0}\right)}\right. \\
\left.\quad+F\left(z_{n} q\left(\delta_{0}, d_{0}\right)+\overline{z_{n}} \overline{q\left(\delta_{0}, d_{0}\right)}, \delta_{0}, d_{0}\right)\right\rangle \\
=\frac{\sqrt{3} i-1}{2} z_{n}+\left\langle p\left(\delta_{0}, d_{0}\right),\right. \\
\left.\quad F\left(z_{n} q\left(\delta_{0}, d_{0}\right)+\overline{z_{n}} \overline{q\left(\delta_{0}, d_{0}\right)}, \delta_{0}, d_{0}\right)\right\rangle .
\end{aligned}
$$

After calculation, we can choose

$$
\begin{gathered}
\left(\delta_{0}, \frac{\sqrt{3} i-3}{2}-\delta_{0} a_{11}\right)^{T}, \\
\left(\frac{\sqrt{3} i\left(3+2 \delta_{0} a_{11}\right)+3}{6 \delta_{0}}, \frac{\sqrt{3} i}{3}\right)^{T}
\end{gathered}
$$

as $q\left(\delta_{0}, d_{0}\right)$ and $p\left(\delta_{0}, d_{0}\right)$, respectively. form

By (26), map (15) can be transformed into the complex

$$
z \longmapsto \frac{\sqrt{3} i-1}{2} z+\sum_{2 \leq k+l \leq 3} \frac{1}{k ! l !} g_{k l}\left(\delta_{0}, d_{0}\right) z^{k} \bar{z}^{l},
$$

where

$$
\begin{aligned}
g_{20} & =g_{11}=g_{02} \\
& =\delta_{0}^{2} a_{13}+\frac{\sqrt{3}}{3} \delta_{0}^{2} i\left(2 \delta_{0} d-3 a_{13}-2 \delta_{0} a_{11} a_{13}\right), \\
g_{30} & =g_{21}=g_{12}=g_{03} \\
& =-3 \delta_{0}^{3} a+\sqrt{3} \delta_{0}^{3} a i\left(3+2 \delta_{0} a_{11}\right) .
\end{aligned}
$$


Here, we denote $g_{k l}\left(\delta_{0}, d_{0}\right)$ by $g_{k l}$, with $k+l=2,3$. And $h_{k l}\left(\delta_{0}, d_{0}\right)$ would be denoted by $h_{k l}$, with $k+l=2,3$ in the introduced transformation.

Now, we introduce the following transformation to annihilate some second order terms:

$$
z=\omega+\frac{1}{2} h_{20} \omega^{2}+h_{11} \omega \bar{\omega}+\frac{1}{2} h_{20} \bar{\omega}^{2},
$$

where coefficients $h_{k l}$ with $k+l=2$ will be confirmed in the following, and we can obtain

$$
\begin{aligned}
\omega= & z-\frac{1}{2} h_{20} z^{2}-h_{11} z \bar{z}-\frac{1}{2} h_{20} \bar{z}^{2}+\frac{1}{2}\left(h_{20}^{2}+h_{11} \bar{h}_{02}\right) z^{3} \\
& +\left(\frac{3}{2} h_{20} h_{11}+\left|h_{11}\right|^{2}+\left|h_{02}\right|^{2}\right) z^{2} \bar{z} \\
& +\left(\frac{1}{2} h_{11} \bar{h}_{20}+h_{11}^{2}+h_{02} \bar{h}_{11}+\frac{1}{2} h_{20} h_{02}\right) z \bar{z}^{2} \\
& +\frac{1}{2}\left(h_{02} \bar{h}_{20}+h_{11} h_{02}\right) \bar{z}^{3}+O\left(|z|^{4}\right) .
\end{aligned}
$$

Thus, using (30) and its inverse transformation, map (28) is changed into the following form:

$$
\omega \longmapsto \frac{\sqrt{3} i-1}{2} \omega+\sum_{2 \leq k+l \leq 3} \frac{1}{k ! l !} \varrho_{k l} \omega^{k} \vec{\omega}^{l}+O\left(|\omega|^{4}\right),
$$

where

$$
\begin{aligned}
\varrho_{20}= & g_{20}+\sqrt{3} h_{20} i, \\
\varrho_{11}= & 2 g_{11}+(\sqrt{3} i-3) h_{11}, \\
\varrho_{02}= & g_{02}, \\
\varrho_{30}= & \frac{3}{2}(3-\sqrt{3} i) h_{20} g_{20}+3 g_{11} \bar{h}_{02} \\
& -\frac{3}{2}(\sqrt{3} i-1) h_{11} \bar{g}_{02}+\frac{3}{2}(\sqrt{3} i+3) h_{20}^{2}+g_{30}, \\
\varrho_{21}= & \frac{1}{2}(5+\sqrt{3} i) h_{11} g_{20}+(2-\sqrt{3} i) h_{20} g_{11} \\
& +2 \bar{h}_{11} g_{11}+(1-\sqrt{3} i) h_{11} \bar{g}_{11}+g_{02} \bar{h}_{02} \\
& +\frac{1}{2}(1+\sqrt{3} i) h_{02} \bar{g}_{02}+g_{21} \\
& +\frac{1}{2}(5 \sqrt{3} i-3) h_{11} h_{20}+(\sqrt{3} i-3) \bar{h}_{11} h_{11}, \\
\varrho_{12}= & g_{20} h_{02}+\frac{1}{2}(1-\sqrt{3} i) h_{11} \bar{g}_{20} \\
& +\left(\bar{h}_{20}+(\sqrt{3} i+3) h_{11}\right) g_{11}+(\sqrt{3} i+1) h_{02} \bar{g}_{11}+g_{12} \\
& +\left(\frac{1}{2}(1-\sqrt{3} i) h_{20}+2 \bar{h}_{11}\right) g_{02} \\
& -\frac{1}{2}(3+\sqrt{3} i) h_{11} \bar{h}_{20}-(3+\sqrt{3} i) h_{11}^{2}-2 \sqrt{3} i h_{02} \bar{h}_{11},
\end{aligned}
$$

$$
\begin{aligned}
\varrho_{03}= & \frac{3}{2}(1+\sqrt{3} i) h_{02} \bar{g}_{20}+3 g_{11} h_{02}+3 \bar{h}_{20} g_{02} \\
& +\frac{3}{2}(1+\sqrt{3} i) h_{11} g_{02}+g_{03}+\frac{3}{2}(3-\sqrt{3} i) h_{02} \bar{h}_{20} .
\end{aligned}
$$

By setting

$$
h_{20}=\frac{\sqrt{3} i}{3} g_{20}, \quad h_{11}=\frac{3+\sqrt{3} i}{6} g_{11}, \quad h_{02}=0,
$$

then we have $\varrho_{20}=\varrho_{11}=0, \varrho_{02}=g_{02}$ and $\varrho_{30}, \varrho_{21}, \varrho_{12}, \varrho_{03}$ can be simplified in the following. Hence, the transformation (30) is defined and

$$
\begin{aligned}
\varrho_{30}= & \frac{3-\sqrt{3} i}{2} g_{11} \bar{g}_{02}+\sqrt{3} i g_{20}^{2}+g_{30}, \\
\varrho_{21}= & \frac{3+2 \sqrt{3} i}{3} g_{20} g_{11}+\frac{3-\sqrt{3} i}{3}\left|g_{11}\right|^{2}+g_{21}, \\
\varrho_{12}= & \frac{3+\sqrt{3} i}{6} g_{20} g_{02}+\frac{3-\sqrt{3} i}{3} \bar{g}_{11} g_{02} \\
& +\frac{3+\sqrt{3} i}{3} g_{11}^{2}-\frac{\sqrt{3} i}{3} \bar{g}_{20} g_{11}+g_{12}, \\
\varrho_{03}= & \sqrt{3} i g_{11} g_{02}-\sqrt{3} i \bar{g}_{20} g_{02}+g_{03} .
\end{aligned}
$$

To further simplify map (32), we introduce the following transformation:

$$
\omega=\zeta+\frac{1}{6} h_{30} \zeta^{3}+\frac{1}{2} h_{12} \zeta \bar{\zeta}^{2}+\frac{1}{2} h_{21} \zeta^{2} \bar{\zeta}+\frac{1}{6} h_{03} \bar{\zeta}^{3} .
$$

After using (36) and its inverse transformation, map (32) is changed into the following form:

$$
\zeta \longmapsto \frac{\sqrt{3} i-1}{2} \zeta+\frac{g_{02}}{2} \bar{\zeta}^{2}+\sum_{k+l=3} \frac{1}{k ! l !} \widetilde{\varrho}_{k l} \zeta^{k} \bar{\zeta}^{l}+O\left(|\zeta|^{4}\right),
$$

where

$$
\begin{gathered}
\widetilde{\varrho}_{30}=\varrho_{30}+\frac{\sqrt{3} i-3}{2} h_{30}, \quad \widetilde{\varrho}_{21}=\varrho_{21}, \\
\widetilde{\varrho}_{12}=\varrho_{12}+\sqrt{3} i h_{12}, \quad \widetilde{\varrho}_{03}=\varrho_{03}+\frac{\sqrt{3} i-3}{2} h_{03} .
\end{gathered}
$$

By setting

$$
\begin{gathered}
h_{30}=\frac{3+\sqrt{3} i}{6} \varrho_{30}, \quad h_{21}=0, \quad h_{12}=\frac{\sqrt{3} i}{3} \varrho_{12}, \\
h_{03}=\frac{3+\sqrt{3} i}{6} \varrho_{03},
\end{gathered}
$$

then we have $\widetilde{\varrho}_{30}=\widetilde{\varrho}_{21}=\widetilde{\varrho}_{03}=0$. Hence, the transformation (36) is defined. Using transformation (36), map (32) finally becomes the following normal form of the bifurcation with $1: 3$ resonance:

$$
\zeta \longmapsto \frac{\sqrt{3} i-1}{2} \zeta+\widetilde{B}\left(\delta_{0}, d_{0}\right) \bar{\zeta}^{2}+\widetilde{C}\left(\delta_{0}, d_{0}\right) \zeta|\zeta|^{2}+O\left(|\zeta|^{4}\right)
$$




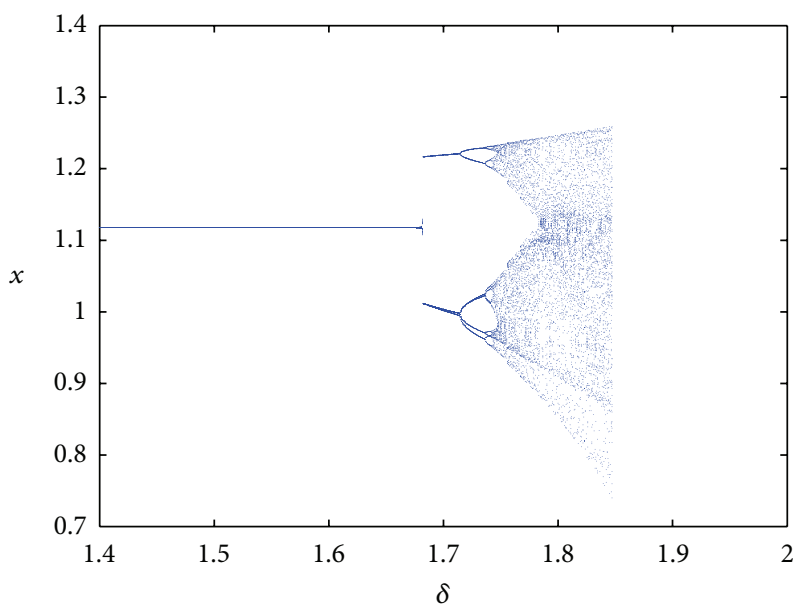

(a)

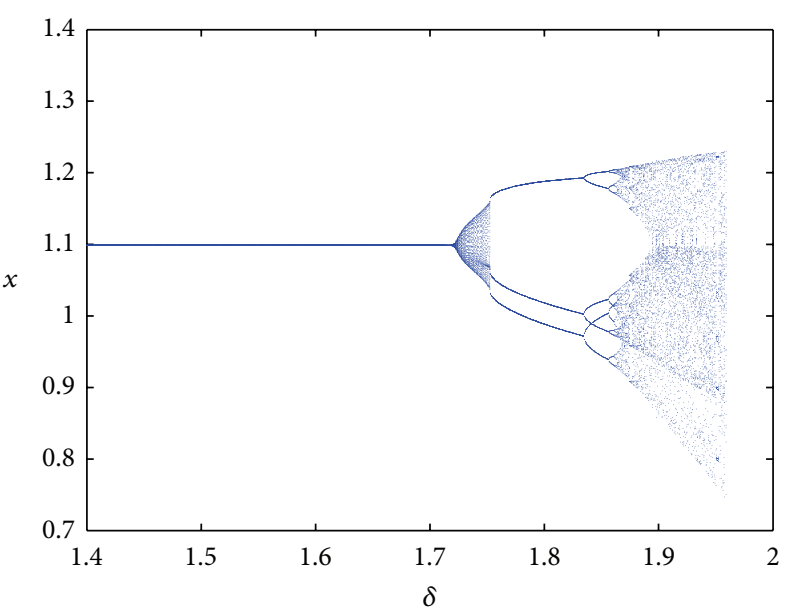

(b)

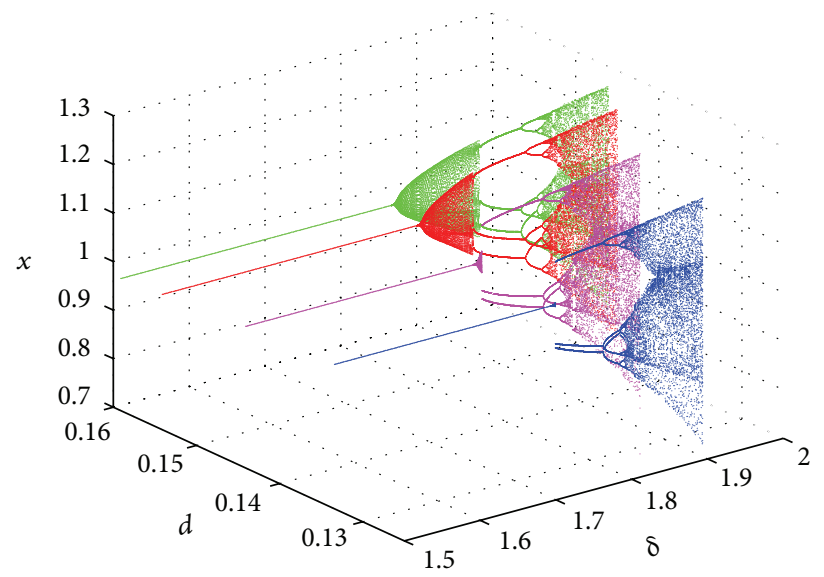

(c)

FIGURE 1: $1: 3$ resonance bifurcation diagram at $E$ with $a=2, b=3, c=0.8, d=0.12428002$, and $\delta=1.6771238$. (a) In $(\delta, x)$ plane with $d=0.12428002$. (b) In $(\delta, x)$ plane with $d=0.14$. (c) In $(\delta, d, x)$ plane.

where

$$
\begin{gathered}
B\left(\delta_{0}, d_{0}\right)=\frac{g_{02}}{2} \\
C\left(\delta_{0}, d_{0}\right)=\frac{g_{20} g_{11}(3+2 \sqrt{3} i)}{6}+\frac{(3-\sqrt{3} i)\left|g_{11}\right|^{2}}{6}+\frac{g_{21}}{2} .
\end{gathered}
$$

If $B_{1}\left(\delta_{0}, d_{0}\right)=-(3 / 2)(\sqrt{3} i+1) B\left(\delta_{0}, d_{0}\right), C_{1}\left(\delta_{0}, d_{0}\right)=$ $-3\left|B\left(\delta_{0}, d_{0}\right)\right|^{2}-(3(1+\sqrt{3} i) / 2) C\left(\delta_{0}, d_{0}\right)$, a similar argument as in Lemma 9.13 in [2] can be obtained.

Theorem 2. Let $\left(\delta_{0}, d_{0}\right) \in F$. If $B_{1}\left(\delta_{0}, d_{0}\right) \neq 0$ and $\operatorname{Re}\left(C_{1}\left(\delta_{0}, d_{0}\right)\right) \neq 0$, then map (3) has the the following complex dynamical behaviors:

(a) there is a Neimark-Sacker bifurcation curve at the trivial fixed point $E_{0}$ of map (40);

(b) there is a saddle cycle of period-three corresponding to the saddle fixed point $E_{k}(k=1,2,3)$ of map (40); (c) there is a homoclinic structure formed by the stable and unstable invariant manifolds of the period-three cycle intersecting transversally in an exponentially narrow parameter region.

Remark 3. Here, the intersection of these manifolds, which form a homoclinic tangency, implies the existence of Smale horseshoes and therefore an infinite number of long-period orbits appear. It illustrates a route from period-3 to chaos.

\section{Numerical Simulations}

In this section, the 2-dimensional and 3-dimensional bifurcation diagrams show that the $1: 3$ resonance is the degenerate case of Neimark-Sacker bifurcation. So there exists a closed invariant circle near the 1:3 resonance. Here, we provide the following case to illustrate the dynamic behaviors of map (3).

Take parameters $a=2, b=3, c=0.8, d=d_{0}=$ 0.12428002 , and $\delta=\delta_{0}=1.6771238$ in map (3). We know that map (3) has a fixed point $E(1.1176267,-0.95523687)$. The eigenvalues of the corresponding Jacobian matrix $J(E)$ 


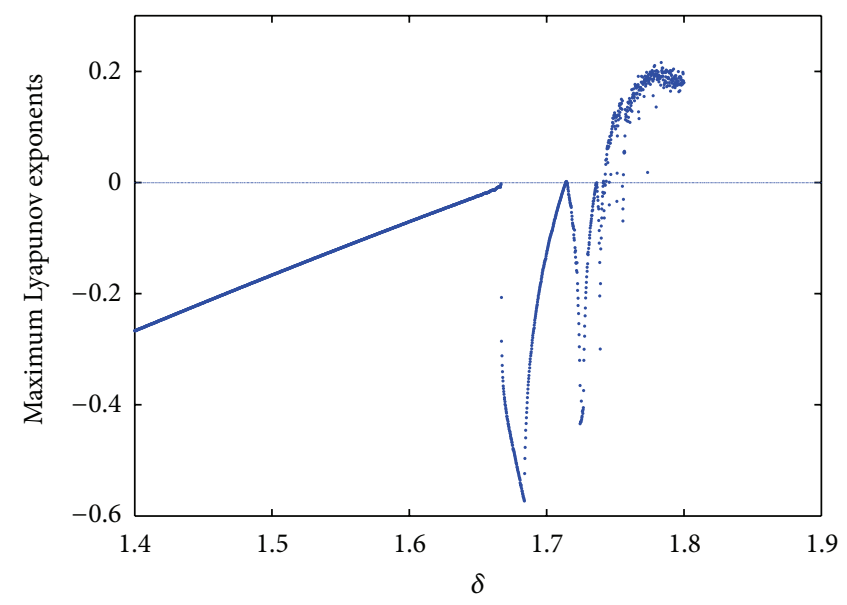

(a)

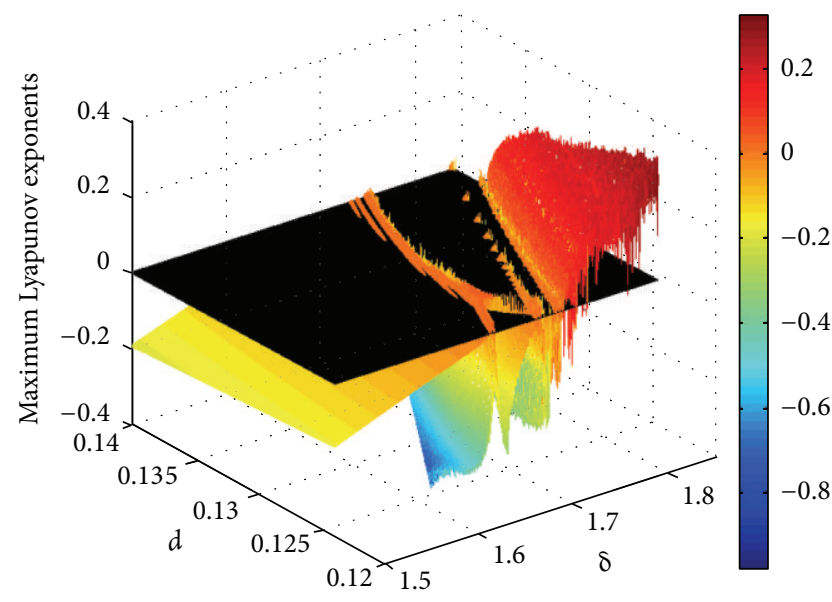

(c)

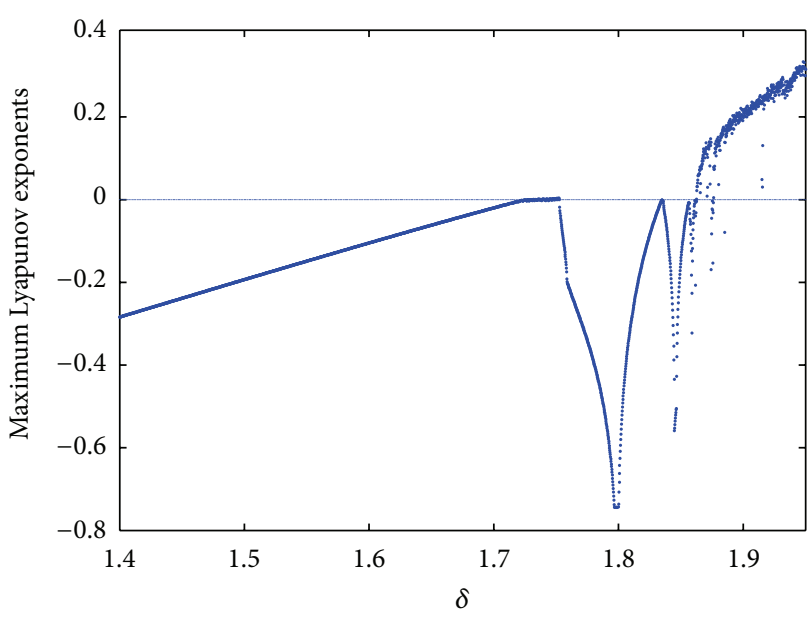

(b)

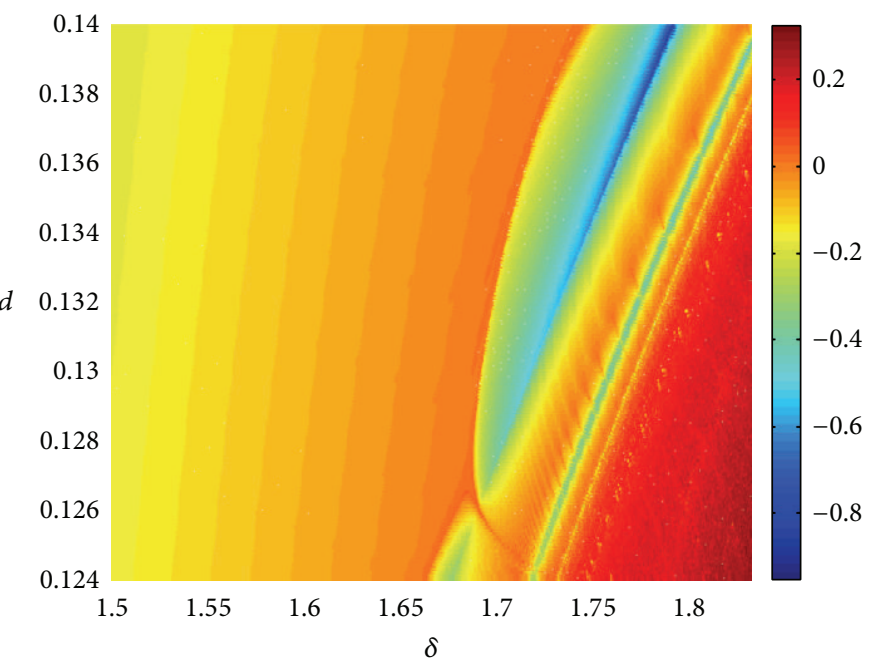

(d)

Figure 2: Maximum Lyapunov exponents of map (3) near 1:3 resonance $E$ as $\delta$ and $d$ vary. (a) and (b) are maximum Lyapunov exponents corresponding to (a) and (b) in Figure 1. (c) and (d) are 3-dimensional maximum Lyapunov exponents in $[1.5,1.82] \times[0.124,0.14]$ and 2-dimensional projection onto $(\delta, d)$.

are $\lambda_{1,2}= \pm 0.8660254347 i-0.5000000165 \approx( \pm \sqrt{3} i-$ $1) / 2$. After calculating, we further have $\operatorname{Re}\left(C_{1}\left(\delta_{0}, d_{0}\right)\right)=$ $-284.6634292 \neq 0$. Therefore, from Theorem 2 , we see that fixed point $E$ is a $1: 3$ resonance point.

Figures 1(a) and 1(b) show the 2-dimensional bifurcation diagrams when $d=0.12428002$ and $d=0.14$, respectively, and $\delta$ varies in a neighborhood of $\delta_{0}=1.6771238$. Figure 1(c) shows the 3-dimensional bifurcation diagram when $\delta, d$ vary in a neighborhood of $\left(\delta_{0}, d_{0}\right)=(1.6771238,0.12428002)$. From Figure 1(c), we can observe the relations between $1: 3$ resonance and Neimark-Sacker bifurcation. In fact, the $1: 3$ resonance is the degenerate case of Neimark-Sacker bifurcation when $\theta_{0}=2 \pi / 3$. Here, $e^{ \pm i \theta_{0}}$ is the eigenvalues of the Jacobian matrix (6). Moreover, the flip bifurcation occurs after the Neimark-Sacker bifurcation and 1:3 resonance. The Lyapunov exponents corresponding to the bifurcation diagram in Figure 1 are computed and plotted in Figure 2. We easily see that there are the positive Lyapunov exponents and negative Lyapunov exponents. It means that map (3) has chaotic and periodic behaviors near the 1:3 resonance. The 3-dimensional maximum Lyapunov exponents are given in Figure 2(c).

Figures 3(a)-3(o) show the phase portraits of map (3) near $E$ for different $\delta$ and $d$. Furthermore, as $d$ varies around $d=d_{0}=0.12428002$ and $\delta_{0}=1.6771238$, from Figures $3(\mathrm{a})-3(\mathrm{~g})$, we can see that period -3 orbits and period -6 orbits, eventually leading to chaos when $d$ decreases to 0.11325 . This is the classical route to chaos. Besides, as $\delta$ increases and $d=d_{0}=0.12428002$ from Figures $3(\mathrm{~h})-3(\mathrm{j})$, we can observe that there exists a fixed point connecting to three saddles, chaos, and more new complex phenomena in certain regions near E. Here, in Figure 3(k), the different colours are chosen to demonstrate the different orbits near the $1: 3$ resonance, which shows the homoclinic structure near a $1: 3$ resonance. Furthermore, both Smale horseshoes and an infinite number of long-period orbits occur. Finally, from Figures 3(m)-3(o), 


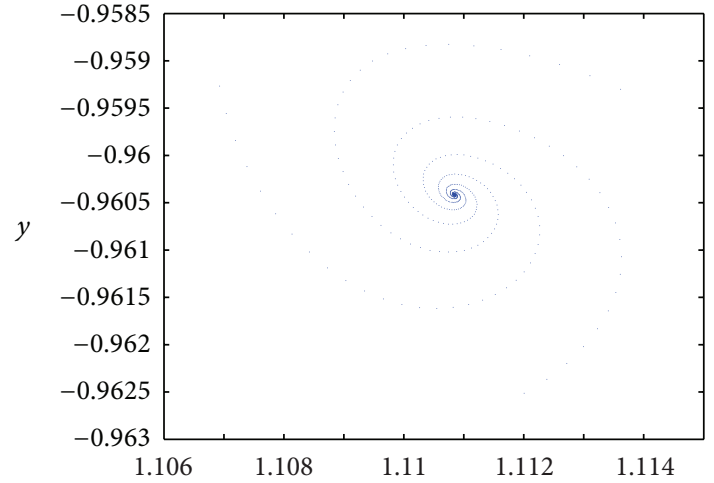

(a)

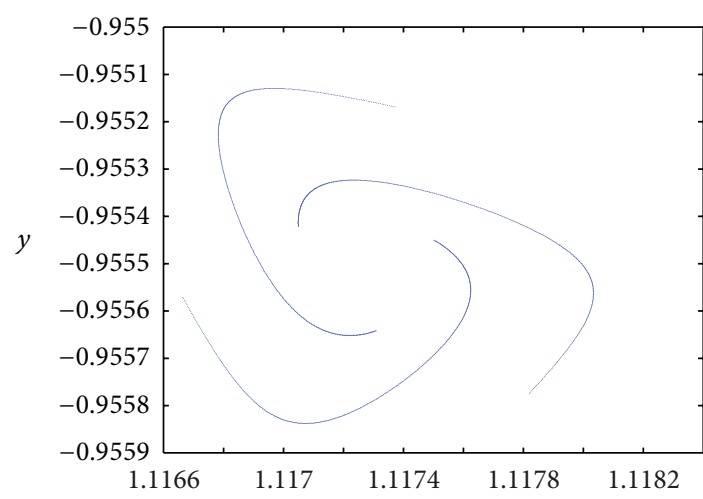

(c)

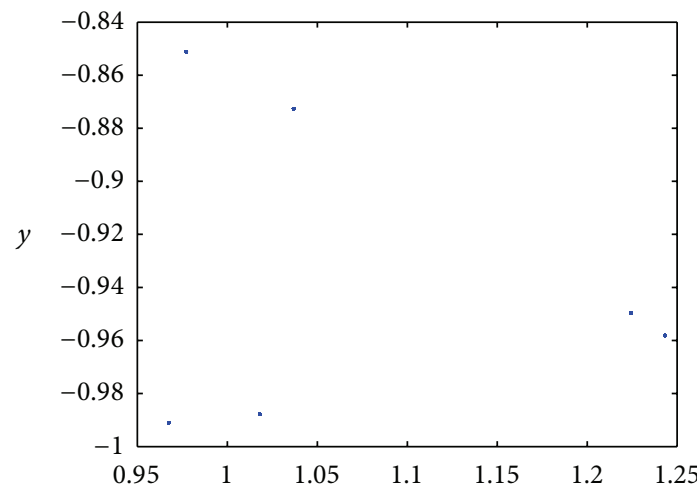

(e)

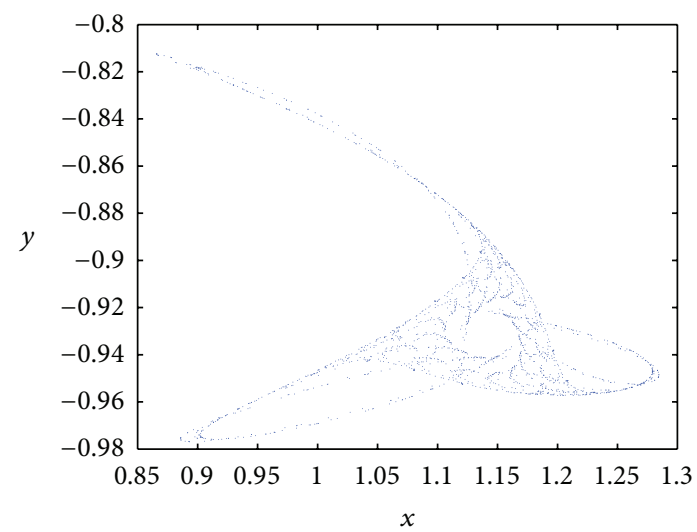

(g)

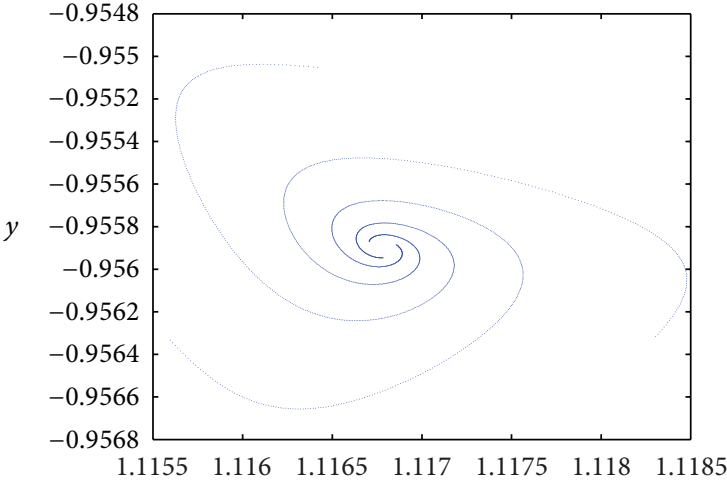

(b)

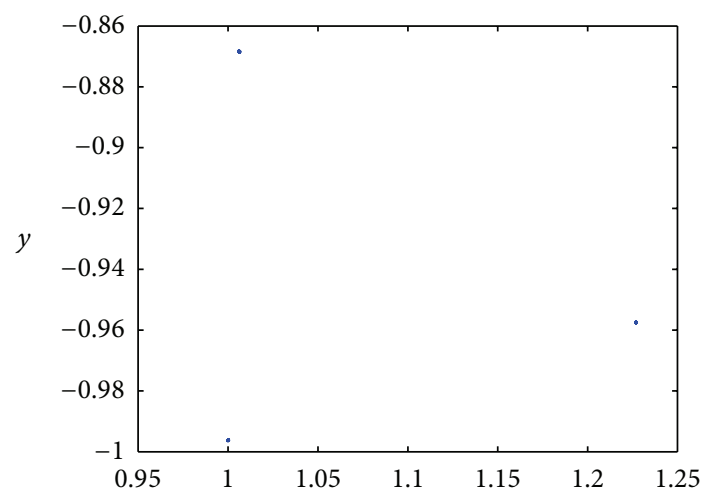

(d)

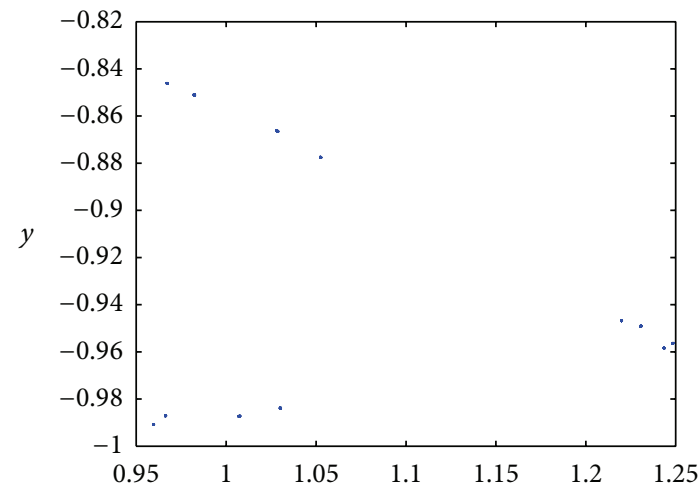

(f)

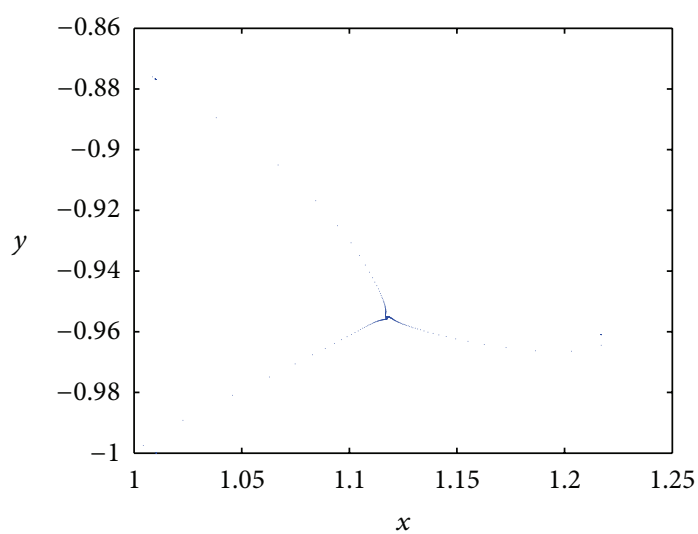

(h)

Figure 3: Continued. 


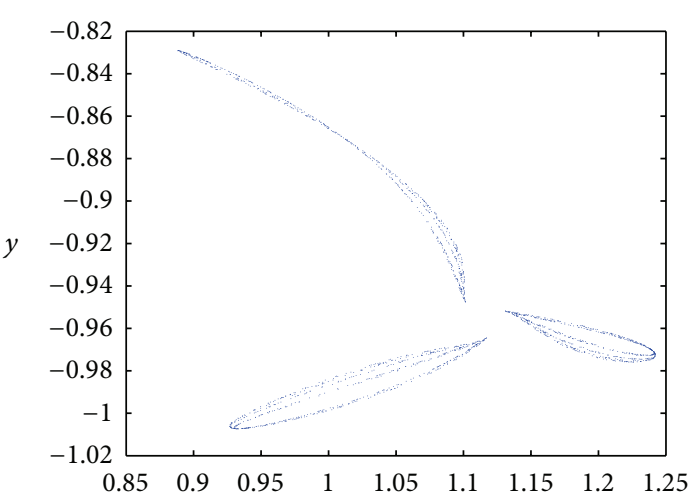

(i)

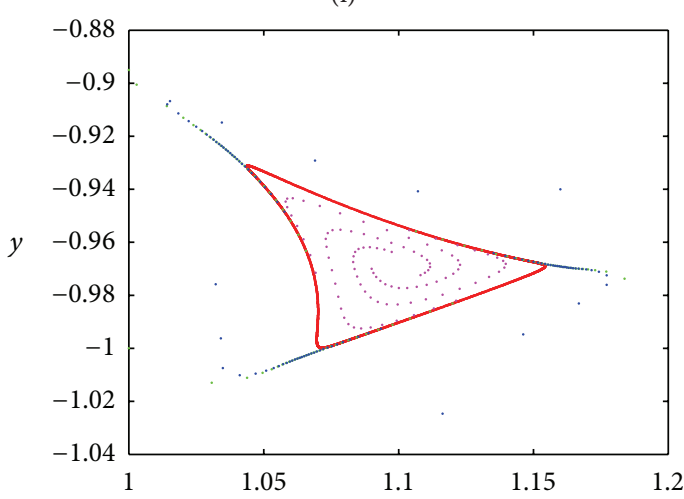

(k)

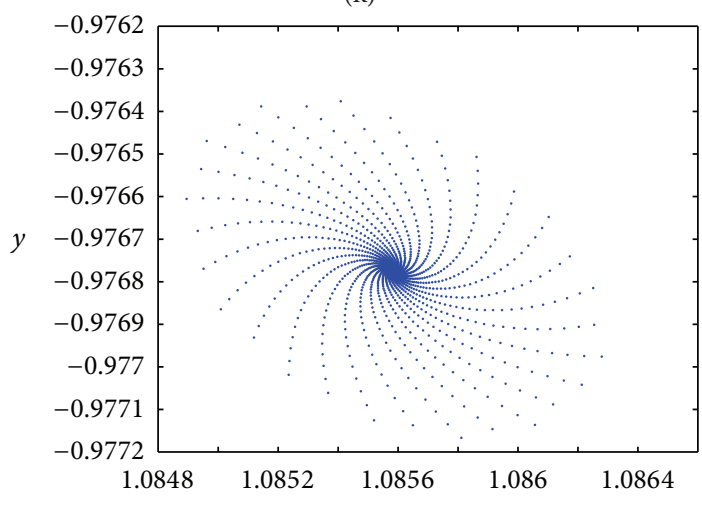

(m)

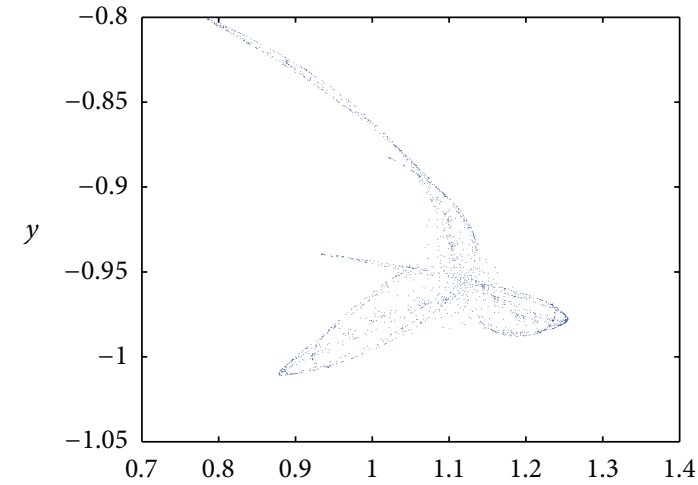

(j)

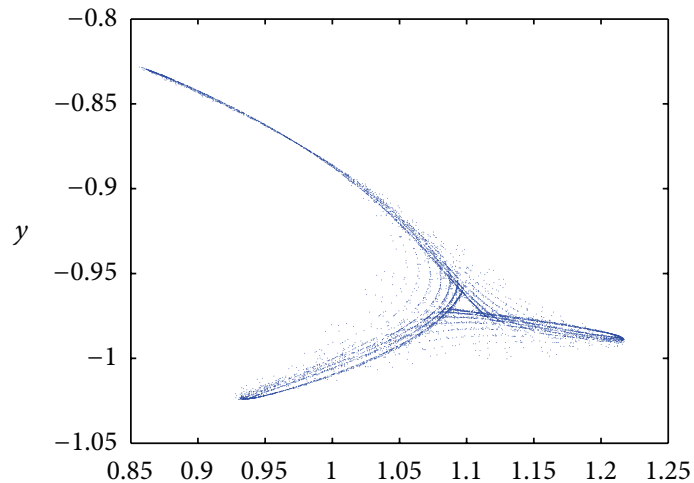

(l)

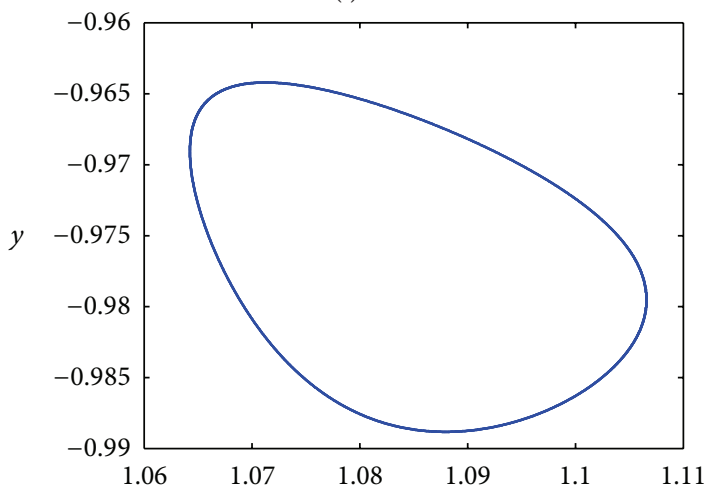

(n)

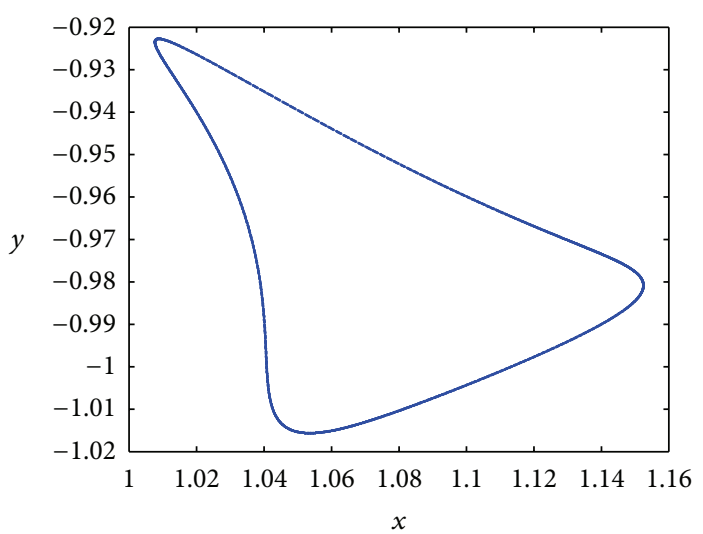

(o)

Figure 3: Phase portraits corresponding to Figure 1 for different $\delta$ and $d$. (a) $\delta=1.6771238, d=0.13$; (b) $\delta=1.6771238$, $d=0.125$; (c) $\delta=1.6771238, d=0.12457$; (d) $\delta=1.6771238, d=0.12$; (e) $\delta=1.6771238, d=0.115$; (f) $\delta=1.6771238, d=0.1134$; (g) $\delta=1.6771238$, $d=0.09$; (h) $\delta=1.685, d=0.12428002$; (i) $\delta=1.78, d=0.12428002$; (j) $\delta=1.83, d=0.12428002$; (k) $\delta=1.75, d=0.14$; (l) $\delta=1.9$, $d=0.14 ;(\mathrm{m}) \delta=1.76, d=0.15 ;(\mathrm{n}) \delta=1.77, d=0.15$; (o) $\delta=1.83, d=0.15$. 
the phase portraits show that Neimark-Sacker bifurcation occurs.

\section{Conclusion}

In this paper, we investigated the $1: 3$ resonance of a discrete Hindmarsh-Rose model. Here, we examined the fixed points of the model in detail and showed that the model exhibits the $1: 3$ resonance. Furthermore, near 1:3 resonance point, the Neimark-Sacker bifurcation and the homoclinic bifurcation can occur. The onset of 1:3 resonance means that, in some cases, there is a region such that the model will have an invariant circle from three-saddle cycle.

Here, we want to note that $1: 3$ resonance involves the bifurcations of $\mathbb{Z}_{3}$ symmetric system, which are not discussed in this paper. From the presented phase portraits, some symmetric phenomena can be observed. The homoclinic loop can explain the transition between spiking and bursting.

\section{Conflict of Interests}

The authors declare that there is no conflict of interests regarding the publication of this paper.

\section{Acknowledgments}

The authors thank the editor and the referees for their valuable suggestions and comments which led to the improvement of the paper.

\section{References}

[1] J. Guckenheimer and P. Holmes, Nonlinear Oscillations, Dynamical Systems, and Bifurcations of Vector Fields, Springer, Berlin, Germany, 1983.

[2] Y. A. Kuznetsov, Elements of Applied Bifurcation Theory, Springer, New York, NY, USA, 3rd edition, 2004.

[3] S. Wiggins, Introduction to Applied Nonlinear Dynamical Systems and Chaos, vol. 2, Springer, New York, NY, USA, 2nd edition, 2003.

[4] J. L. Hindmarsh and R. M. Rose, "A model of the nerve impulse using two first-order differential equations," Nature, vol. 296, no. 5853, pp. 162-164, 1982.

[5] R. FitzHugh, "Impulses and physiological state in theoretical models of nerve membrane," Biophysical Journal, vol. 1, pp. 445467, 1961.

[6] J. Nagumo, S. Arimoto, and S. Yoshizawa, "An active pulse transmission line simulating nerve axon," Proceedings of the IRE, vol. 50, pp. 2061-2070, 1962.

[7] J. M. Gonzalez-Miranda, "Complex bifurcation structures in the Hindmarsh-Rose neuron model," International Journal of Bifurcation and Chaos, vol. 17, no. 9, pp. 3071-3083, 2007.

[8] X. Liu and S. Liu, "Codimension-two bifurcation analysis in two-dimensional Hindmarsh-Rose model," Nonlinear Dynamics, vol. 67, no. 1, pp. 847-857, 2012.

[9] M. Storace, D. Linaro, and E. de Lange, "The HindmarshRose neuron model: bifurcation analysis and piecewise-linear approximations," Chaos, vol. 18, no. 3, Article ID 033128, 2008.
[10] S. Nikolov, "An alternative bifurcation analysis of the RoseHindmarsh model," Chaos, Solitons and Fractals, vol. 23, no. 5, pp. 1643-1649, 2005.

[11] S. Tsuji, T. Ueta, H. Kawakami, H. Fujii, and K. Aihara, "Bifurcations in two-dimensional Hindmarsh-Rose type model," International Journal of Bifurcation and Chaos, vol. 17, no. 3, pp. 985998, 2007.

[12] D. Linaro, A. Champneys, M. Desroches, and M. Storace, "Codimension-two homoclinic bifurcations underlying spike adding in the Hindmarsh-Rose burster," SIAM Journal on Applied Dynamical Systems, vol. 11, no. 3, pp. 939-962, 2012.

[13] A. Shilnikov and M. Kolomiets, "Methods of the qualitative theory for the Hindmarsh-Rose model: a case study. A tutorial," International Journal of Bifurcation and Chaos, vol. 18, no. 8, pp. 2141-2168, 2008.

[14] Q. Chen, Z. Teng, L. Wang, and H. Jiang, "The existence of codimension-two bifurcation in a discrete SIS epidemic model with standard incidence," Nonlinear Dynamics. An International Journal of Nonlinear Dynamics and Chaos in Engineering Systems, vol. 71, no. 1-2, pp. 55-73, 2013.

[15] Z. He and X. Lai, "Bifurcation and chaotic behavior of a discretetime predator-prey system," Nonlinear Analysis. Real World Applications, vol. 12, no. 1, pp. 403-417, 2011.

[16] Z. He and J. Qiu, "Neimark-Sacker bifurcation of a third-order rational difference equation," Journal of Difference Equations and Applications, vol. 19, no. 9, pp. 1513-1522, 2013.

[17] J. L. Hindmarsh and R. M. Rose, "A model of neuronal bursting using three coupled first order differential equations," Proceedings of the Royal Society of London Series B: Biological sciences, vol. 221, no. 1222, pp. 87-102, 1984.

[18] A. L. Hodgkin and A. F. Huxley, "A quantitative description of membrane current and its application to conduction and excitation in nerve," The Journal of Physiology, vol. 117, no. 4, pp. 500-544, 1952.

[19] G. Innocenti, A. Morelli, R. Genesio, and A. Torcini, "Dynamical phases of the Hindmarsh-Rose neuronal model: studies of the transition from bursting to spiking chaos," Chaos, vol. 17, no. 4, Article ID 043128, 2007.

[20] Z. Jing, Y. Chang, and B. Guo, "Bifurcation and chaos in discrete FitzHugh-Nagumo system," Chaos, Solitons \& Fractals, vol. 21, no. 3, pp. 701-720, 2004.

[21] B. Li and Z. He, "Bifurcations and chaos in a two-dimensional discrete Hindmarsh-Rose model," Nonlinear Dynamics, vol. 76, no. 1, pp. 697-715, 2014

[22] X. Liu and D. Xiao, "Complex dynamic behaviors of a discretetime predator-prey system," Chaos, Solitons \& Fractals, vol. 32, no. 1, pp. 80-94, 2007.

[23] S.-S. Chen, C.-Y. Cheng, and Y.-R. Lin, "Application of a two-dimensional Hindmarsh-Rose type model for bifurcation analysis," International Journal of Bifurcation and Chaos in Applied Sciences and Engineering, vol. 23, no. 3, Article ID 1350055, 2013.

[24] S. Ma and Z. Feng, "Fold-Hopf bifurcations of the RoseHindmarsh model with time delay," International Journal of Bifurcation and Chaos, vol. 21, no. 2, pp. 437-452, 2011.

[25] D. Terman, "The transition from bursting to continuous spiking in excitable membrane models," Journal of Nonlinear Science, vol. 2, no. 2, pp. 135-182, 1992.

[26] X.-J. Wang, "Genesis of bursting oscillations in the HindmarshRose model and homoclinicity to a chaotic saddle," Physica D: Nonlinear Phenomena, vol. 62, no. 1-4, pp. 263-274, 1993. 
[27] B. Li and Z. M. He, "1:2 and 1:4 resonances in a twodimensional discrete Hindmarsh-Rose model," Nonlinear Dynamics, 2014.

[28] A. D. Polyanin and A. I. Chernoutsan, A Concise Handbook of Mathematics, Physics, and Engineering Science, CRC Press, New York, NY, USA, 2011. 


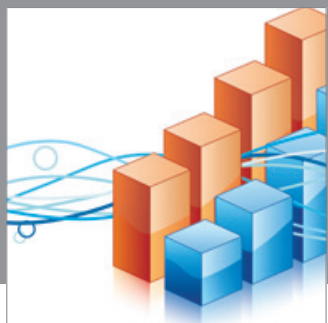

Advances in

Operations Research

mansans

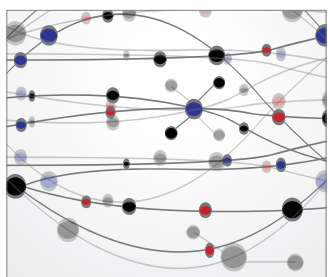

The Scientific World Journal
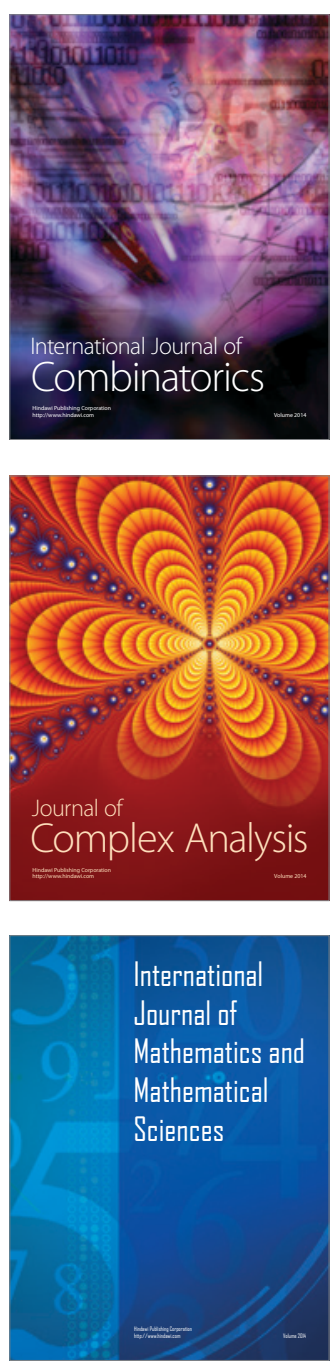
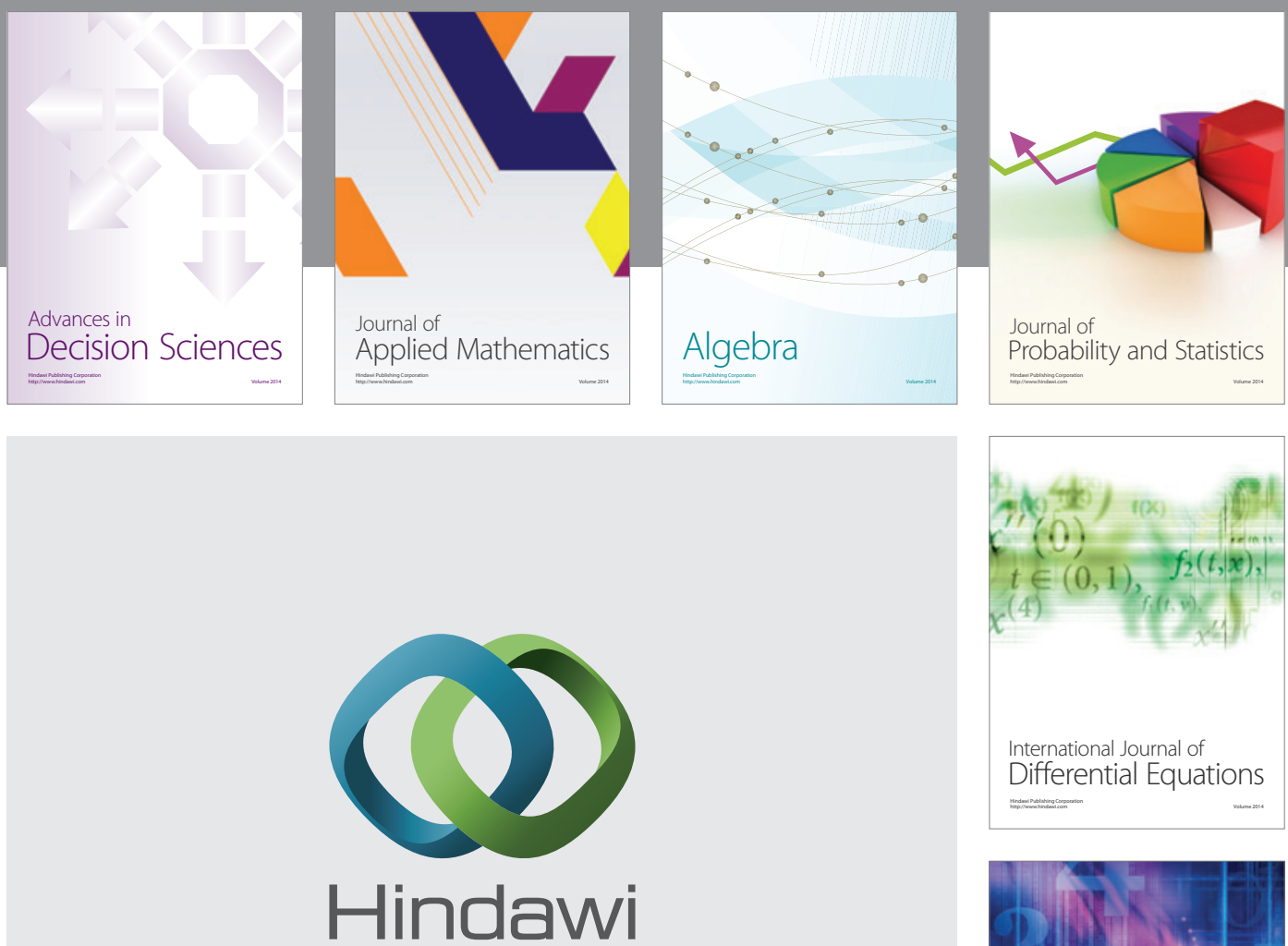

Submit your manuscripts at http://www.hindawi.com
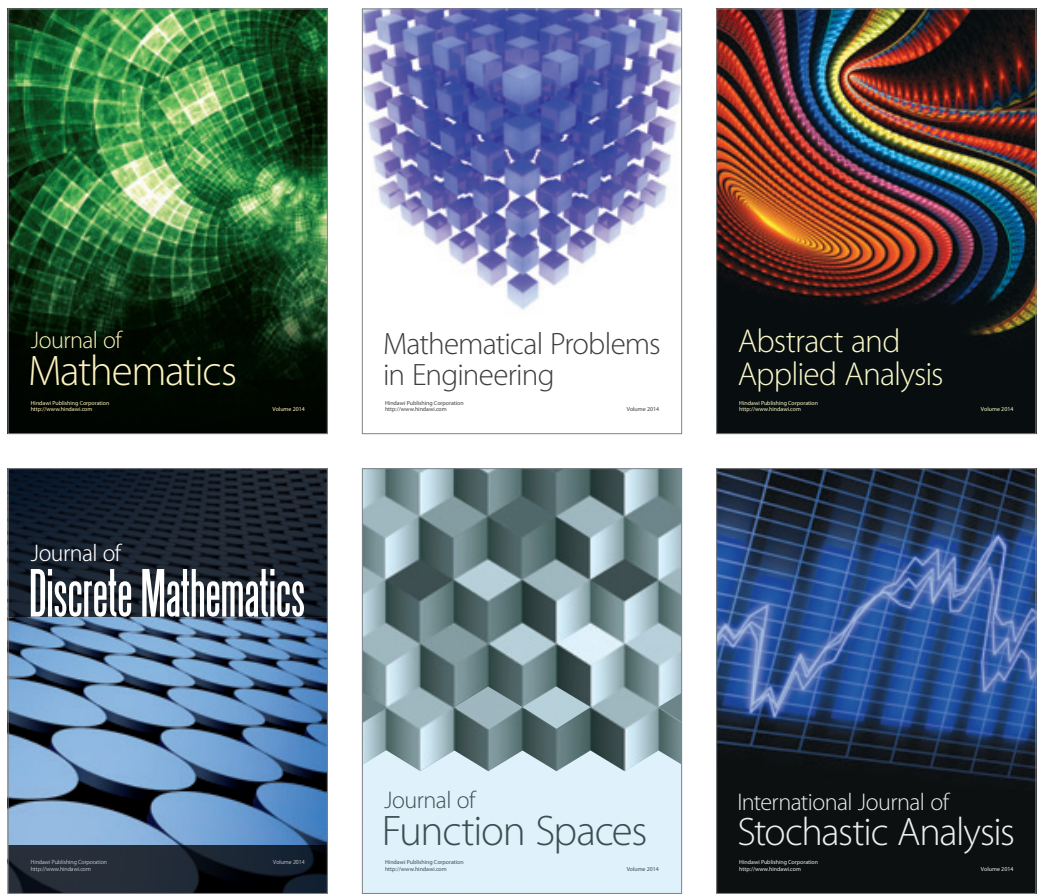

Journal of

Function Spaces

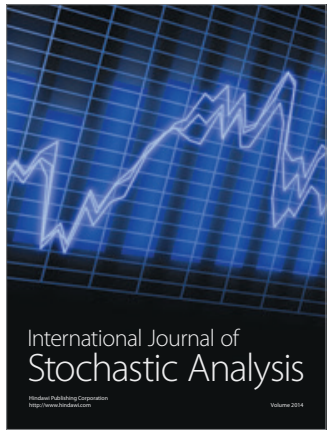

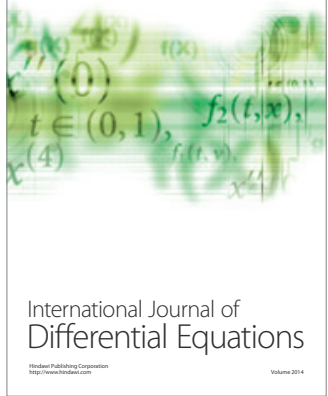
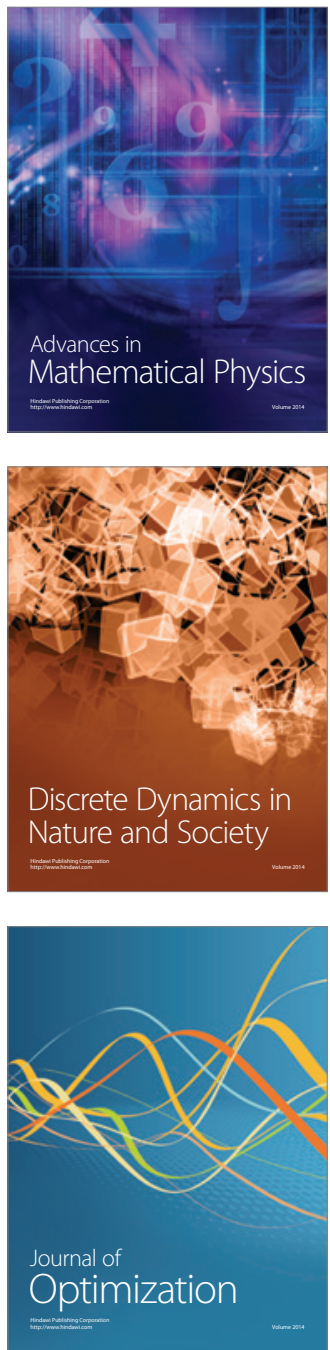